\title{
Modal Location-Resource-Process Logic for Access Control
}

\author{
David Pym \\ Computing Science \\ University of Aberdeen
Aberdeen. Scotland. U.K. \\ d.j.pym@abdn.ac.uk
}

The logic MBI is a substructural modal logic of locations, resources, and processes that is closely related to both the bunched logic BI and Hennessy-Milner logic. MBI stands in a (slightly weak) Hennessy-Milner correspondence with the synchronous process calculus of located resources, LSCRP. Starting from motivations in large-scale systems modelling, I will introduce MBI and LSCRP, and explain how they can be used to address some theoretical and practical questions in access control. 